\title{
Pulmonary venous atrial obstruction after the Senning procedure: relief by catheter balloon dilatation
}

\author{
John D Coulson, Rufus B Jennings Jr, David H Johnson
}

\begin{abstract}
Pulmonary venous atrial stenosis developed after a modified Senning procedure in an infant with transposition of the great arteries. The pulmonary venous atrium was surgically revised, but the obstruction recurred. The stenosis was then enlarged by percutaneous balloon catheter dilatation. There was an immediate haemodynamic and echocardiographic improvement. Clinical and echocardiographic improvement persisted nine months after dilatation. The procedure was complicated by staphylococcal endarteritis.
\end{abstract}

\section{Case report}

Division of Pediatric Cardiology, Department of Pediatrics, Eastern Virginia Medical School, Children's Hospital of The King's Daughters, and Sentara Norfolk General Hospital, Norfolk, Virginia, USA

$\mathrm{J} D$ Coulson

R B Jennings Jr

D H Johnson

Correspondence to Dr John D Coulson, Department of Pediatrics, Children's Hospital of Th King's Daughters, 800 W Olney Raughters, 800 Virginia 23507, USA.
A 16 month old female infant presented with dyspnoea. She was born with transposition of the great arteries and had a Senning procedure performed at the age of three months. Her pulmonary venous atrium was augmented with bovine pericardium. She did well until she was eight months old when pulmonary Reoperation disclosed stenosis of the pulmonary venous atrium by neointimal tissue associated with the bovine pericardial patch. This tissue and the patch were resected, and the remaining defect was closed with native pericardium. Postoperatively, clinical and echocardiographic evaluation indicated resolution of the obstruction. Dyspnoea recurred when she was 16 months old and a new $3 / 6$ venous atrial obstruction rapidly appeared.

Haemodynamic and echocardiographic data

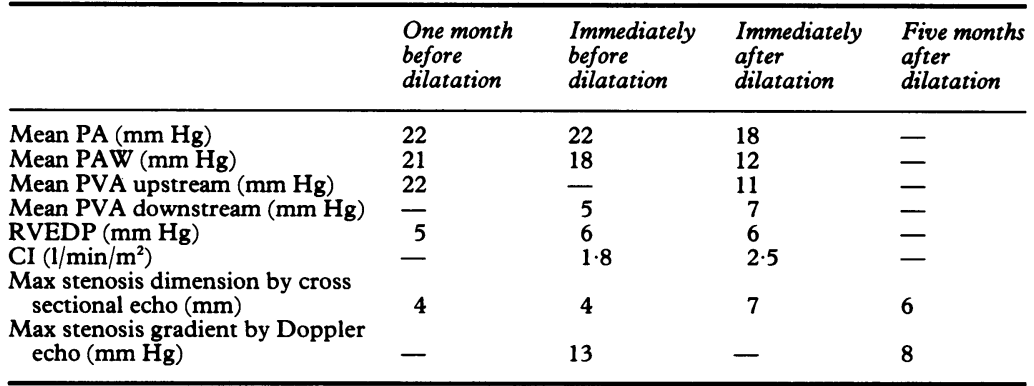

$\mathrm{CI}$, cardiac index, mean of 6-7 thermodilution determinations; PA, pulmonary artery pressure; PAW, pulmonary artery wedge pressure; PVA, pulmonary venous atrium pressure; RVEDP, right ventricular end diastolic pressure.

right ventricular end diastolic pressure.
Pressures grouped by time are representative of multiple samples and were generally not Pressures grouped by time
measured simultaneously.

continuous murmur was heard over the sternum. Cross sectional and Doppler echocardiography and cardiac catheterisation showed severe obstruction within the pulmonary venous atrium (table). Reoperation was considered, but our cardiovascular surgeons opted to try balloon dilatation of the obstruction. This approach was offered to the patient's parents as an alternative though untested treatment, and they gave their informed consent.

The procedure was performed on 17 May 1989. The patient was 18 months old and she weighed $10.6 \mathrm{~kg}$. The cardiac index was measured by the thermodilution technique (table). Right femoral arterial pressures were continuously monitored via the sidearm of a $6 \mathrm{~F}$ sheath. We used serial measurement of activated coagulation times (Hemochron, International Technidyne Corporation, Edison, NJ, USA) to assess heparinisation. A $3.6 \mathrm{~F}$ end hole catheter with a tip angled at approximately $90^{\circ}$ (White preformed (renal) catheter, Becton, Dickinson and Company, Rutherford, NJ, USA) was positioned in the right ventricle. The tip of this catheter was turned to the patient's right, to direct a 0.018 inch flexible and steerable guide wire (HiTorque Floppy, Advanced Cardiovascular Systems, Temecula, Ca, USA) across the tricuspid valve into the pulmonary venous atrium. The catheter was advanced into the atrium, and the tip was turned posteriorly to direct the guide wire through the stenosis in the pulmonary venous atrium to the lower left pulmonary vein. The catheter was then advanced to this position. The guide wire was exchanged for a 0.021 inch guide wire, and the catheter was exchanged for an angiographic catheter. The pressure drop across the stenosis was measured (table) and pulmonary venous angiography was performed.

A $5 \mathrm{~F}$ angioplasty catheter with a $5 \mathrm{~mm}$ diameter $\times 2 \mathrm{~cm}$ long balloon (polyethylene Dotter balloon dilatation catheter, Cook, Bloomington, In, USA) was advanced over the larger guide wire, purged with carbon dioxide, filled with dilute contrast material in the descending aorta, and positioned across the stenosis. Maximum balloon inflation produced no haemodynamic relief. Therefore, an identical catheter was similarly positioned across the stenosis via the left femoral artery. 
Figure 1 Two angioplasty balloons simultaneously inflated in the stenotic segment of the pulmonary venous atrium nearly abolishing the waist produced by the stenosis. A thermodilution catheter is positioned in the right pulmonary artery.

(A) Posteroanterior and

(B) lateral angiograms.

Figure 2 Lateral angiogram of the stenotic segment of the pulmonary venous atrium after balloon dilatation showing a tear in the inferior margin of the segment (arrow).

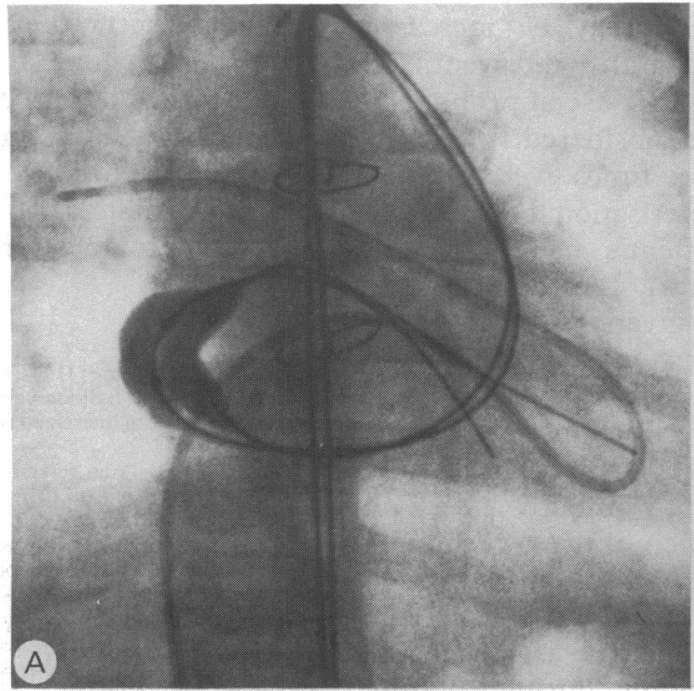

Because the left internal iliac artery was stenotic and would not accommodate a $6 \mathrm{~F}$ sheath, the second catheter was introduced directly through the skin. Four simultaneous double balloon inflations were performed (fig 1). The angioplasty balloon catheters were withdrawn and measurements of haemodynamic function (table) and pulmonary venous angiography (fig 2) were repeated before the end of the procedure.

The next day the patient was afebrile and seemed well. Her catheterisation wounds were clear and her femoral and pedal pulses were normal bilaterally. Cross sectional echocardiography showed relief of the stenosis in the pulmonary venous atrium (table). The patient was discharged from the hospital, but signs of infection in her left leg appeared over the next three days. She was readmitted to hospital with staphylococcal endarteritis of the left external iliac artery from which she recovered

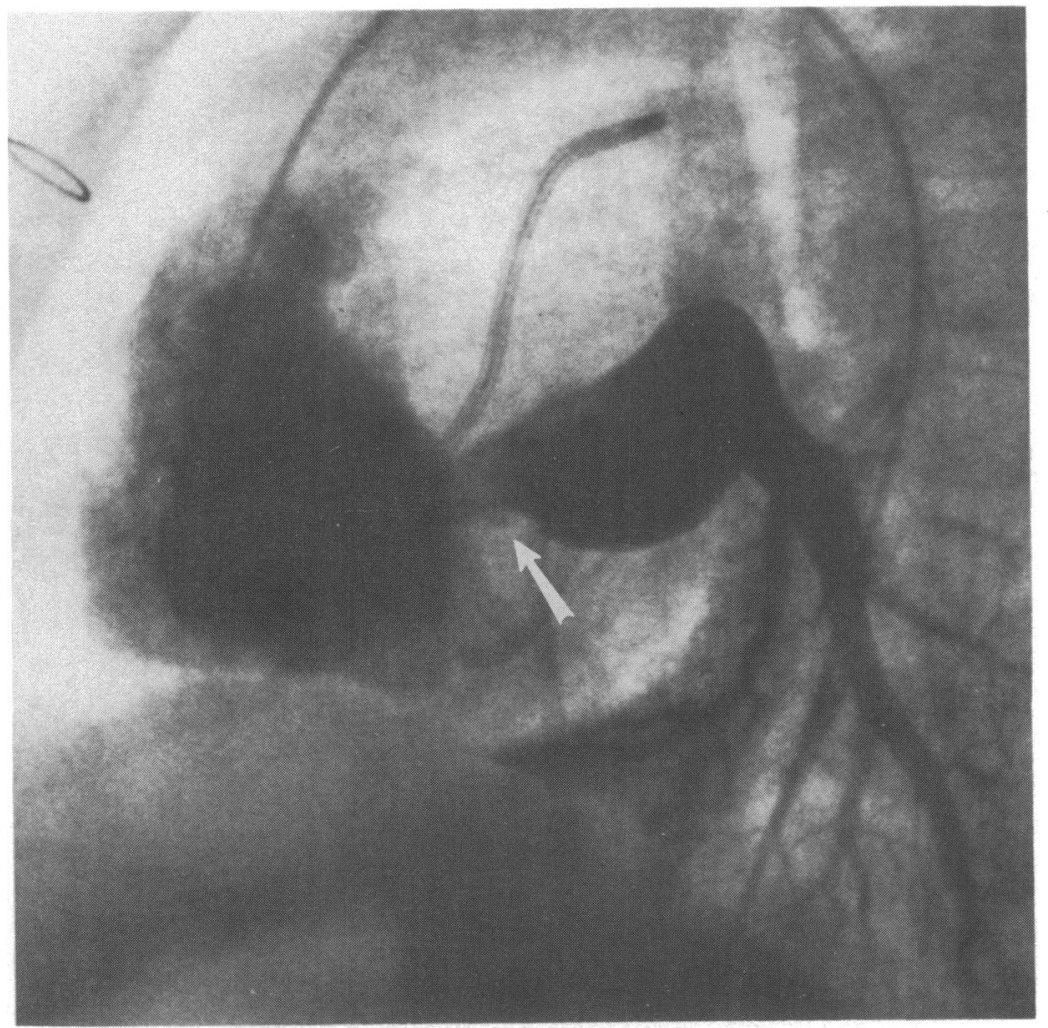

after a complicated course. Five months after the procedure, cross sectional and Doppler echocardiography showed persistent relief of the stenosis (table). Nine months after the procedure the patient remained symptom free.

\section{Discussion}

It is important to select a balloon with a large enough diameter to dilate the stenosis. In our patient, the echocardiographic maximum dimension of the stenosis and its angiographic vertical extent were $4 \mathrm{~mm}$. We chose to dilate the stenosis with $2-5 \mathrm{~mm}$ diameter balloons, the effective diameter of which was $8.2 \mathrm{~mm}^{12}$ This combination of balloons, the largest low profile balloons then available to us, did not fully abolish the stenosis.

However, the pressure drop was halved and the cardiac index increased. Furthermore, angiography showed a tear in the inferior wall of the stenosis, and echocardiography showed relief of the narrowing. Subsequent clinical and echocardiographic evaluations showed persistence of the improvement. A balloon diameter three to four times larger than the echocardiographically measured narrowest dimension of the pulmonary venous pathway obstructed after the Mustard operation was recommended for dilating such obstructions. ${ }^{3}$ Thus larger balloons might have been more effective in our patient. Use of two small balloons seems preferable to a single large balloon technique because small balloons may be more easily manoeuvred through the systemic circulation, and the risk of mechanical injury to the femoral arteries or heart may be reduced.

After the procedure staphylococcal endarteritis and thrombosis developed in our patient's previously stenosed left external iliac artery. Ileofemoral arteriography showed a well developed collateral circulation to the left leg which may have masked the thrombosis initially. The artery was surgically explored, and thrombectomy was performed. Septic embolisation produced osteomyelitis in the femur, septic arthritis in the knee, and necrosis of part of the great toe. Neither endocarditis nor meningitis developed. This complication, unique in our experience and associated with no recognised technical mishap, indicates that 
risk of serious infection may be increased during complex and lengthy interventional catheterisations and that the use of prophylactic antibiotics should be considered.

Activated coagulation times were serially determined in the catheterisation laboratory to assess rapidly the patient's need for supplemental doses of heparin. ${ }^{45}$ At the outset 100 units/kg of heparin was given. Six supplementary doses of 50 units/kg were given to maintain activated coagulation times of between 312 and 410 seconds. Guide wires remained within the systemic arterial circulation for several hours. However, only a trace of thrombus was seen on one of these wires when it was eventually withdrawn, and no other thrombus was seen during the procedure. Thus we believe that the activated coagulation time is a valuable measure of heparinisation during lengthy interventional catheterisations in infants and children.
Our experience reported in an infant with pulmonary venous atrial obstruction after a modified Senning procedure and that reported in children with similar stenoses after the Mustard operation ${ }^{3}$ indicate that balloon catheter dilatation of these obstructions is a feasible alternative to reoperation.

1 Radtke W, Keane JF, Fellows KE, Lang P, Lock JE. Percutaneous balloon valvotomy of congenital pulmonary stenosis using oversized balloons. J Am Coll Cardiol 1986; 8:909-15.

2 Yeager SB. Balloon selection for double balloon valvotomy [Letter]. J Am Coll Cardiol 1987;9:467-8.

3 Cooper SG, Sullivan ID, Bull C, Taylor JFN. Balloon dilatation of pulmonary venous pathway obstruction after dilatation of pulmonary venous pathway obstruction after Mustard repair for transpositio

4 Scott JA, Berenstein A, Blumenthal D. Use of the activated coagulation time as a measure of anticoagulation during interventional procedures. Radiology 1986;158:849-50.

5 Ogilby JD, Kopelman HA, Klein LW, Agarwal JB. Adequate heparinization during PTCA: assessment using activated clotting time [Abstract]. J Am Coll Cardiol 1988;11:237A 